Upper Gl Surgery, Liverpool Heart and Chest Hospital NHS Foundation Trust, Liverpool, UK

Introduction Surgical resection is considered the gold standard curative treatment for oesophageal cancer, however, there is debate regarding the role of endoscopic resection for "early" (Tis, T1) oesophageal cancer. However, studies have identified that a significant number of early cancers will have lymph node metastases (LNM) at time of diagnosis. The aim of this study was to determine the incidence of LNM in early oesophageal cancer patients in a 10 year UK cohort.

Methods A total of 482 consecutive patients who had transthoracic oesophagectomy for adenocarcinoma or squamous cell carcinoma, at a supra-regional upper GI cancer centre, were registered on a prospective database between 2002 and 2012. Patients with early oesophageal cancer were retrieved from the database based on pre-operative endoscopic and radiological staging.

Results Of the 482 patients, $53(11 \%)$ had early oesophageal cancer, of which $41(77 \%)$ were adenocarcinoma, 11 (21\%) squamous cell carcinoma and $1(2 \%)$ carcinoma in situ. The median lymph node harvest was 14 (IOR 9-17). Lymph node metastases were present in $4(7.6 \%)$ cases, all of which had tumour extending into the submucosal layer.

Conclusion Early oesophageal cancer has a significant risk of lymph node involvement especially when the submucosal layer is breached by tumour. In our opinion, surgical resection remains the gold standard to achieve cure in patients with early cancer.

Competing interests None declared.

\section{PWE-041 OBESITY IS ASSOCIATED WITH IMPROVED SURVIVAL IN SURGICALLY RESECTED OESOPHAGEAL CANCER}

\section{doi:10.1136/gutjnl-2012-302514d.41}

${ }^{1} \mathrm{~T}$ S Athwal, ${ }^{* 1} \mathrm{~S}$ Love, ${ }^{1} \mathrm{~S}$ Bruce, ${ }^{2} \mathrm{R}$ Page, ${ }^{1} \mathrm{M}$ Hartley, ${ }^{1} \mathrm{~N}$ Howes. ${ }^{1}$ Upper Gl Surgery, Liverpool Heart and Chest Hospital NHS Foundation Trust, Liverpool, UK; ${ }^{2}$ Thoracic \& Upper GI Surgery, Liverpool Heart and Chest Hospital NHS Foundation Trust, Liverpool, UK

Introduction Obesity is an increasing health burden in the western world and a known risk factor for oesophageal cancer. The aim of this study was to determine the impact of obesity on circumferential resection margin (CRM), lymph node yield (LNY) and survival among a 10 year cohort of surgically resected oesophageal cancer patients.

Methods All patients who underwent transthoracic oesophagectomy for histologically proven adenocarcinoma or squamous cell carcinoma, performed at a supra-regional upper GI cancer centre, were registered on a prospective database between 2002 and 2012. Total LNY, CRM and survival was compared between different BMI groups: normal (18.5-24.9), overweight (25-30), and obese $(>30)$. Patients who were malnourished prior to surgery were excluded from the analysis.

Results A total of 482 consecutive patients underwent oesphagectomy during the study period, of which the complete dataset was available for 441 (91\%); normal (155), overweight (177) and obese (91). The median lymph node yield was 16 (IOR 11-22), normal 15 (10-23), overweight $17(12-23)$ and obese 15 (10-21), with no significant difference seen between the three groups $\left(p=0.4565, \chi^{2}\right.$ test). The CRM was involved in $30(27 \%)$ of the obese patients compared to 55 (31\%), 54 (35\%) and of overweight, healthy patients respectively ( $p=0.0615, \chi^{2}$ test).

Conclusion Obesity appears to confer a survival advantage in patients undergoing surgical resection for oesophageal cancer. Interestingly, there appeared to be a trend towards an uninvolved CRM in obese patients but no significant impact on the lymph node yield.

Competing interests None declared.

\section{PWE-042 THE RELEVANCE OF THE SIEWERT CLASSIFICATION IN THE ERA OF MULTIMODAL THERAPY FOR ADENOCARCINOMA OF THE GASTRO-OESOPHAGEAL JUNCTION}

doi:10.1136/gutjnl-2012-302514d.42

1,2 F Noble, ${ }^{2}$ S B Bailey, ${ }^{2} \mathrm{~J} J$ Kelly, ${ }^{2} \mathrm{~J}$ P Byrne, ${ }^{1,2} \mathrm{~T}$ J Underwood. ${ }^{1}$ Cancer Sciences Unit, Faculty of Medicine, University of Southampton, Southampton, UK; ${ }^{2}$ Department of Surgery, University Hospital Southampton Foundation Trust, Southampton, UK

Introduction Since the early 1980s the Siewert classification has been used to plan treatment for tumours of the gastro-oesophageal junction. However, the relationship between tumour site and survival has not been conclusively demonstrated, with conflicting outcomes in the largest series, before the widespread application of neoadjuvant chemotherapy. The aim of this study was to evaluate whether there were differences in the biology and clinical characteristics of adenocarcinomas by Siewert type, in a contemporary cohort of patients, in whom the majority had received neoadjuvant chemotherapy. The relationship of the surgical approach and tumour site with patient survival was also assessed.

Methods A prospective database was reviewed for all patients who underwent resection for adenocarcinoma of the distal oesophagus and gastro-oesophageal junction from 2005 to 2011. In our unit, based on pre-operative assessment, distal oesophageal, type I and II tumours are treated as oesophageal cancer, with transthoracic procedures. Type III tumours are treated as gastric cancer with an abdominal approach. Classification systems used for analysis included TNM 7 for staging, Clavien-Dindo for grading complications and Siewert with final tumour site determined from the pathological specimen. Survival was estimated by Kaplan-Meier analysis excluding inpatient deaths $(n=4)$ and $R 1$ resections $(n=42)$. Results 216 patients underwent oesophagogastric resection: 133 for type I, 51 for type II and 33 for type III tumours. Median follow-up was 2.94 years. $62.5 \%$ of patients received neoadjuvant chemotherapy with no difference between groups. There were no significant differences in age, sex, $\mathrm{pT}$ stage, $\mathrm{pN}$ stage, $\mathrm{pM}$ stage, ASA, or inpatient complications between patients with adenocarcinoma based on their Siewert classification. Type I tumours were significantly associated with coexisting Barrett's metaplasia (presence of Barrett's: Type I 58.3\%, Type II 21.6\%, Type III 9.1\%; pType II > Type I). Median overall survival was significantly shorter for more distal tumours (Type I: 4.96 years vs Type II: 3.3 years vs Type III: 2.64 years; $p=0.04)$. The surgical approach did not influence survival for all tumour types and had no impact on the rate or severity of complications.

Conclusion This study demonstrates significant differences in the biological characteristics of adenocarcinomas of the gastro-oesophageal junction based on their anatomical topographical sub-classification. In the era of multimodal therapy overall survival is worse for tumours arising at or below the gastro-oesophageal junction compared with oesophageal tumours.

Competing interests None declared.

\section{PWE-043 CHANGING EPIDEMIOLOGY OF FOOD BOLUS IMPACTION IS EOSINOPHILIC OESOPHAGITIS TO BLAME?}

doi:10.1136/gutjnl-2012-302514d.43

V Mahesh, ${ }^{*}$ R Holloway, $Q$ N Nguyen. Department of Gastroenterology, Royal Adelaide Hospital, Adelaide, Australia

Introduction The epidemiology of acute food bolus impaction (FBI) of the oesophagus in adults remains unclear. The incidence of eosinophilic oesophagitis (EE) is increasing and is a well-known cause for FBI. Currently, there are no data on the epidemiological changes in FBI and its relationship to EE. 
Aim To evaluate the changes in prevalence and aetiology of FBI of the oesophagus.

Methods Details relating to all patients who presented with FBI to the Department of Gastroenterology at the Royal Adelaide Hospital from 1996 to 2010 were reviewed from a prospective database. The periods were divided into 1996-2000, 2001-2005 and 2006-2010. Detailed endoscopic and histological findings were examined for patients who presented between these periods. Data from coding department were analysed to assess the pattern of presentation and specialties involved.

Results Over 15 years, 248 patients presented with FBI (74\% male) to the Gastroenterology department. The prevalence of FBI increased overtime (1996-2000: $n=30, \quad 2001-2005: n=80$; 2006-2010: $n=137)$. While there was no change in gender, the age of presentation was significantly younger in 2006-2010 (56.2 $\pm 1.6 \mathrm{yrs})$ than that from 2001 to 2005 (61.6 $\pm 1.9 \mathrm{yrs}, \mathrm{p}=0.03)$ and $1996-2000(62.8 \pm 3.3 \mathrm{yrs}, \mathrm{p}<0.01)$. The predominant causes for FBI between 1996 and 2000 related to benign strictures and complications of reflux disease (64\%), and no patient had EE. The diagnosis of EE was suspected during endoscopy in $10 \%$ of patients in $2001-2005$ and $35 \%$ of patients in 2006-2010 ( $p<0.01)$. Oesophageal biopsies were taken significantly more frequently in patients who presented with FBI between 2006-2010 (75\%) as compared to those in $2001-2005(47 \%, p<0.01)$ and $1996-2000$ $(12 \%, p<0.001)$. Histologically proven EE was found in $6.2 \%$ of patients in 2001-2005 and 23\% of patients in 2006-2010 ( $p<0.01)$. There were no significant changes in other aetiologies overtime (benign strictures, reflux disease, malignancy, or post-surgical strictures). Endoscopic findings of "normal" esophageal structure or mucosa reduced overtime occurred in $23 \%$ in $1996-2000,17 \%$ in 2001-2005 and 14\% in 2006-2010.

Conclusion The increased prevalence of FBI overtime is associated with an increased prevalence of eosinophilic oesophagitis. While this may be related, increasing awareness about eosinophilic oesophagitis and lower threshold for performing esophageal biopsy are also likely to contribute.

Competing interests None declared.

\section{Neurogastroenterology and motility PWE-044 GASTROINTESTINAL SYMPTOMS IN THE JOINT HYPERMOBILITY SYNDROME}

doi:10.1136/gutjnl-2012-302514d.44

${ }^{1}$ A Fikree, ${ }^{*}{ }^{1} \mathrm{R}$ Aktar, ${ }^{2} \mathrm{R}$ Grahame, ${ }^{1} 0$ Aziz. ${ }^{1}$ Department of Neurogastroenterology, BICMS, University College London, London, UK; ${ }^{2}$ Department of Rheumatology, University College London, London, UK

Introduction The Joint Hypermobility Syndrome (JHS) is a hereditary connective tissue disorder, characterised by hyperflexibility of the skin and joints and musculoskeletal symptoms. A high prevalence of JHS has been found in patients presenting to GI clinics with unexplained symptoms. ${ }^{1}$ However, the range and prevalence of gastrointestinal (GI) symptoms in JHS patients coming from rheumatology clinics has never been adequately characterised or compared to other patient groups. This was our aim.

Methods Multicentre, cross sectional study in new referrals to GI clinics between April 2010 and December 2011. Patients between the ages of 16-70 with a rheumatological diagnosis of JHS were compared to those in whom JHS had been excluded, at their first GI clinic visit. The validated Bowel Disease Questionnaire was completed for GI symptom information. Clinical examination confirmed or excluded the diagnosis of JHS, using the 1998 Brighton criteria. Patients outside the age range or with limited English were excluded. GI symptoms were considered significant if they occurred at least once a week, and were compared between the two groups, adjusting for sex and age. In view of the multiple comparisons, the significance level was set at 0.005 .

Results 413 patients participated: 43 with a previous rheumatological diagnosis of JHS, and 370 in whom JHS had been excluded. There were significantly more females in the JHS group $(95 \%$ vs $54 \%, p=0.000$ ), and they were younger (mean age: $34.6 \pm 10.97$ vs 43.3 $\pm 14.2, p=0.000)$. Abdominal pain was present in all JHS patients and was significantly more likely to be of more than 5 years duration:(OR 4.38 CI 2.1 to 9.1). Other symptoms which were significantly different between the two groups are shown in Abstract PWE-044 table 1. There was no significant difference in the prevalence of constipation (OR 1.1), diarrhoea (OR 0.2), heartburn (OR 1.3), retrosternal chest pain (OR 2), vomiting (OR 1.2), or dyspepsia (OR 2).

Abstract PWE-044 Table 1 Comparison of Gl symptoms

\begin{tabular}{lllll}
\hline Symptom & \% in JHS & $\begin{array}{l}\text { \% in } \\
\text { non-JHS }\end{array}$ & $\begin{array}{l}\text { OR (adj for } \\
\text { age, sex) }\end{array}$ & CI \\
\hline Alternating bowel habit*** & 63 & 26 & 4.6 & 2.3 to 9.2 \\
Globus** $^{*}$ & 47 & 20 & 3.3 & 1.6 to 6.7 \\
Dysphagia** $^{*}$ & 33 & 12 & 3.4 & 1.5 to 7.5 \\
Regurgitation $^{*}$ & 34 & 12 & 3.0 & 1.4 to 6.6 \\
Nausea* $^{*}$ & 48 & 20 & 2.6 & 1.3 to 5.2 \\
Postprandial fullness $^{* *}$ & 79 & 43 & 4.1 & 1.9 to 9.2 \\
Early satiety $^{* *}$ & 60 & 29 & 2.9 & 1.5 to 5.8 \\
Bloating*** $^{*}$ & 88 & 47 & 6.3 & 2.3 to 16.9 \\
\hline
\end{tabular}

${ }^{*} \mathrm{p}<0.05,{ }^{* *} \mathrm{p}<0.005,{ }^{* * *} \mathrm{p}<0.001$.

Conclusion This is the first comparative study of GI symptoms in JHS patients. They have significantly more upper GI symptoms, bloating and alternating bowel habit even when adjusting for age and sex. In view of the association between JHS and unexplained GI symptoms, it is important to consider the diagnosis of JHS in patients with intractable functional GI symptoms, to enable multidisciplinary management. Further studies are needed to determine the mechanism of symptoms in these patients.

Competing interests None declared.

\section{REFERENCE}

1. Zarate N, Farmer AD, Grahame R, et al. Unexplained gastrointestinal symptoms and joint hypermobility: is connective tissue the missing link? Neurogastroenterol Motil 2009;22:252-e78

\section{PWE-045 GASTROPARESIS - CLASSIFYING SEVERITY AND PREDICTING RESPONSE}

doi:10.1136/gutjnl-2012-302514d.45

B Hudson, ${ }^{*}$ F Fayyaz, R Makins. Department of Gastroenterology, Gloucestershire Hospitals NHS Foundation Trust, Cheltenham, UK

Introduction Idiopathic gastroparesis is a chronic disorder of the stomach characterised by delayed gastric emptying in the absence of recognisable causative pathology such as mechanical obstruction or diabetes. Presenting symptoms include early satiety, vomiting and weight loss. Investigation usually includes gastric emptying studies, however significant overlap in gastric emptying times (GET) between normal and abnormal populations can make diagnosis difficult. Using a case series of patients managed at Gloucestershire Hospitals NHS Foundation Trust we explore the relationship between symptomatic presentation and GET, and attempt to correlate factors predicting successful response to treatment.

Methods Patients with clinical features of gastroparesis, and GET of over 100 min on nuclear medicine studies were identified from local radiology and clinic databases. Medical notes were scrutinised for 